\title{
Involvement of Ceramide in Ischemic Tolerance Induced by Preconditioning with Sublethal Oxygen-Glucose Deprivation in Primary Cultured Cortical Neurons of Rats
}

\author{
Mohammad Iqbal Hossain Bhuiyan, ${ }^{a, b}$ Mohammad Nurul Islam, ${ }^{a}$ Seo Yun Jung, ${ }^{c}$ Hye Hyun Yoo, ${ }^{a}$ \\ Yong Sup LEE, ${ }^{c}$ and Changbae JiN ${ }^{*, a}$ \\ ${ }^{a}$ Doping Control Center, Research Coordination Division, Korea Institute of Science and Technology (KIST); P.O. Box \\ 131, Cheongryang, Seoul 130-650, South Korea: ${ }^{b}$ Department of Biomolecular Science, University of Science and \\ Technology (UST); Daejon 305-333, South Korea: and ${ }^{c}$ Department of Pharmaceutical Sciences, College of Pharmacy \\ and Department of Life and Nanopharmaceutical Science, Kyung Hee University; Seoul 130-701, South Korea. \\ Received May 4, 2009; accepted October 5, 2009; published online October 19, 2009
}

\begin{abstract}
The complex molecular cascades of ischemic tolerance in brain cells remain unclear. Recently, sphingolipidrelated metabolite ceramide has been implicated as a second messenger in many biological functions, including neuronal survival and death. The present study, therefore, examined the roles of ceramide (Cer) in ischemic tolerance induced by preconditioning with sublethal oxygen-glucose deprivation (OGD) using primary cultured cortical neurons of rats. Preconditioning of the neurons with sublethal 1-h OGD produced robust neuroprotection against cell death induced by lethal 3-h OGD imposed $12 \mathrm{~h}$ after preconditioning when measured by the MTT assay. Analysis of sphingolipids using LC-MS/MS showed that the ischemic preconditioning resulted in significant increases in the levels of $C_{16: 0} \mathrm{Cer}, C_{18: 0} \mathrm{Cer}, C_{20: 0} \mathrm{Cer}, \mathrm{C}_{24: 0} \mathrm{Cer}, \mathrm{C}_{24: 1} \mathrm{Cer}$ and the total ceramide contents compared with the sham-washed control group. However, sphingomyelin contents were not significantly changed by the ischemic preconditioning, suggesting that ceramides were increased through the de novo synthetic pathway. In the case of severe OGD paradigm, levels of ceramide and sphingomyelin in the lethal OGD group were not significantly different from those of the control group or the lethal OGD group with preconditioning at any time points studied. Treatment with an inhibitor of de novo ceramide synthesis, fumonisin $B_{1}$, during the ischemic preconditioning period completely blocked preconditioning-induced ischemic tolerance. Moreover, application of a non-cytotoxic concentration of exogenous cell-permeable ceramide produced neuroprotection against lethal OGD. The results suggest that ceramides increased by sublethal OGD preconditioning play an important role in induction of ischemic tolerance.
\end{abstract}

Key words ischemic tolerance; ceramide; oxygen-glucose deprivation; preconditioning; cortical neuron

Ischemic preconditioning is an endogenous protective mechanism induced by a brief episode(s) of sublethal ischemia, which has been demonstrated in a variety of organs and in cellular systems including neuronal cells. ${ }^{1)}$ The underlying molecular mechanisms concerning ischemic tolerance, however, are yet to be elucidated. In this regard, oxygen-glucose deprivation (OGD) preconditioning is an elegant in vitro model to study the neuronal endogenous protective mechanisms as well as their signaling mediators. ${ }^{2)}$

Sphingolipids are a class of complex lipids containing an amide-linked fatty acid and long-chain (sphingoid) base that are important structural components of cell membranes. Although sphingolipids have been considered for many years as structural components of cell membranes, it is now acknowledged that they are also involved in many cellular functions, including cell differentiation, growth, senescence, cell survival and death. ${ }^{3,4)}$ The levels of cellular sphingolipids are regulated by various types of stress conditions, including ionizing radiation, heat shock, anti-cancer drugs and hyperosmolarity to name a few. ${ }^{5}$ ) Recently, sphingolipids have been implicated in neurological disease conditions such as Alzheimer's disease, ${ }^{6}$ epilepsy ${ }^{7)}$ and cerebral ischemia. ${ }^{8)} \mathrm{On}$ the other hand, accumulation of ceramide has also been reported after physical exercise, ${ }^{9,10)}$ a well-known preconditioning treatment, which is reported to improve neurological parameters and reduce neuronal damage after stroke. ${ }^{11,12)}$ However, the actual role of ceramide as a neuronal signaling molecule is still unclear. The exogenous application of ceramide has been found to induce apoptosis in a variety of neuronally derived cells. ${ }^{13)}$ On the other hand, the cytoprotective effects of ceramide have also been reported in primary cultures, ${ }^{14)}$ hypoxic injury ${ }^{15)}$ and a hypoxic preconditioning model. ${ }^{16)}$ Casaccia-Bonnefil et al. (1996) found that $10 \mu \mathrm{M}$ of ceramide was detrimental to oligodendrocytes but not harmful to neurons and astrocytes. ${ }^{17)}$ In isolated rat motoneurons, Irie and Hirabayashi (1998) demonstrated that lower concentrations of ceramide increased cell survival, whereas higher concentrations resulted in cell death. ${ }^{18)}$

It can be noted that sphingolipid metabolites, including ceramides, are metabolically inter-convertible and structurally similar, and exist at very low concentrations in cells. Several methods, such as GC, TLC, HPLC, and enzyme- or antibody-based assays, have been used to identify and quantify sphingolipids. But these procedures are insensitive, nonspecific, and/or time consuming. In addition, they can not measure multiple sphingolipids simultaneously. Recently, mass spectrometric methods, especially LC-MS or LC-MS/MS, have facilitated the identification and quantification of subtle changes in specific sphingolipid metabolites in tissues or cell samples to assign their (patho)physiological roles. ${ }^{19)}$ Moreover, this technique is advantageous because it requires a small amount of scarce primary neuron samples.

Growing importance of sphingolipid metabolites as a signaling molecule in (patho)physiological conditions, led us to examine alterations in the levels of different sphingolipids present in primary cultured cortical neurons of rats subjected 
to sublethal and lethal OGD stress using an LC-MS/MS method. In this study, we have identified and quantified different molecular species of ceramides and sphingomyelins, and addressed their involvement in ischemic tolerance induced in primary cultured cortical neurons of rats.

\section{MATERIALS AND METHODS}

Materials $N$-Acetyl-D-sphingosine $\left(\mathrm{C}_{2}\right.$ ceramide $), N$-butanoyl-D-sphingosine $\left(\mathrm{C}_{4 \cdot 0} \mathrm{Cer}\right), \mathrm{N}$-palmitoyl-D-sphingosine $\left(\mathrm{C}_{16: 0} \mathrm{Cer}\right), N$-oleoyl-D-sphingosine $\left(\mathrm{C}_{18: 1} \mathrm{Cer}\right), \mathrm{N}$-arachidoyl-D-sphingosine $\left(\mathrm{C}_{20: 0} \mathrm{Cer}\right), \mathrm{N}$-lignoceroyl-D-sphingosine $\left(\mathrm{C}_{24: 0} \mathrm{Cer}\right), N$-nervonoyl-D-sphingosine $\left(\mathrm{C}_{24: 1} \mathrm{Cer}\right)$ and fumonisin $\mathrm{B}_{1}\left(\mathrm{FB}_{1}\right)$ were purchased from Sigma Chemicals (St. Louis, MO, U.S.A.). $N$-Stearoyl-D-sphingosine $\left(\mathrm{C}_{18: 0} \mathrm{Cer}\right)$ and sphingomyelin (from brain sources) were purchased from Avanti Polar Lipids (Alabaster, AL, U.S.A.). Chloroform, methanol and tetrahydrofuran of HPLC grade were purchased from J. T. Baker (Phillipsburg, NJ, U.S.A.). All other chemicals were of analytical grade. High purity nitrogen (99.999\%) was obtained from Shin Yang Gas (Seoul, Korea). Tissue culture dishes and plates were purchased from SPL (Seoul, Korea). Protein reagents and protein standards were obtained from Bio-Rad Laboratories (Richmond, CA, U.S.A.). Dulbecco's modified Eagle medium (DMEM), Neurobasal medium, supplement B27, supplement B27 without antioxidant, glutamine, penicillin/streptomycin, fetal bovine serum and trypsin were purchased from Gibco BRL (Grand Island, NY, U.S.A.).

Primary Culture of Cortical Neurons of Rats Primary neuronal cultures of cerebral cortex were obtained from Sprague-Dawley rats (Orient, Seoul, Korea), at approximately $17-18$ embryonic days according to the method of Brewer (1995) with some modifications. ${ }^{20)}$ Briefly, cerebral cortices (free of meninges) were pooled, minced and gently dissociated by mechanical trituration in $\mathrm{Ca}^{2+}$ and $\mathrm{Mg}^{2+}$ free Hank's balanced salt solution and incubated with $0.1 \%$ trypsin for $20 \mathrm{~min}$ at $37^{\circ} \mathrm{C}$. Enzymatic digestion was terminated by mixing the suspension with DMEM supplemented with $10 \%$ fetal bovine serum. Cells were passed through nylon mesh and pelleted by brief centrifugation. The dissociated cells were plated on $100-\mathrm{mm}$ dishes (pre-coated with $0.01 \mathrm{mg} / \mathrm{ml}$ poly-L-lysine) at a density of $7.5 \times 10^{6}$ cells $/$ dish in seeding medium consisting of Neurobasal medium supplemented with $2 \%$ B27, $0.5 \mathrm{~mm}$ glutamine, $25 \mu \mathrm{M}$ glutamate, $100 \mathrm{units} / \mathrm{ml}$ penicillin and $100 \mu \mathrm{g} / \mathrm{ml}$ streptomycin. Cultures were maintained at $37{ }^{\circ} \mathrm{C}$ in a humidified incubator containing $5 \% \mathrm{CO}_{2}$ and $95 \%$ air (normoxia). Cultures were refreshed twice a week with maintenance medium (seeding medium without glutamate). This serum-free culture condition allowed us to maintain cultures with a very low percentage $(<5 \%)$ of glia. $^{21)}$

Preconditioning Experiments with Oxygen-Glucose Deprivation Simulated ischemic injury experiments with neuronal cells were performed at $6-8 \mathrm{~d}$ in vitro according to the procedure described by Goldberg and Choi (1993) with little modifications. ${ }^{22)}$ Briefly, at $6-7 \mathrm{~d}$ in vitro, cells were washed twice with glucose-free HEPES-buffered saline (5.4 mм KCl, $51.3 \mathrm{~mm} \mathrm{NaCl}, 20 \mathrm{~mm} N$-(hydroxyethyl)piperazine- $N N^{\prime}$-2-ethanesulfonic acid (HEPES), $1.8 \mathrm{mM} \mathrm{CaCl}_{2}$, $0.01 \mathrm{~mm}$ glycine, $\mathrm{pH}$ 7.3). To initiate OGD, deoxygenated glucose-free HEPES-buffered saline (bubbled with a gas mixture of $95 \% \mathrm{~N}_{2}$ and $5 \% \mathrm{CO}_{2}$ for $30 \mathrm{~min}$ ) was added to cultures and immediately transferred to an anaerobic chamber (PLAS-LABS, Lansing, MI, U.S.A.) which was previously rinsed with an anaerobic gas mixture $\left(5 \% \mathrm{CO}_{2}, 5 \% \mathrm{H}_{2}\right.$ and $90 \% \mathrm{~N}_{2}$ ). Cells were maintained at $37^{\circ} \mathrm{C}$ for appropriate time periods to produce either sublethal $1-\mathrm{h}$ or lethal $3-\mathrm{h}$ OGD. The oxygen concentration was continuously monitored with an anaerobic indicator strip (Oxoid Ltd., Hampshire, England). At the end of the appropriate period, OGD was terminated by changing the deoxygenated HEPES buffer to an ordinary, pre-warmed Neurobasal media containing B27 without antioxidant supplements and returning the cells to a humidified incubator with normoxia. Ischemic neuronal injury was assessed by the MTT (3-(4,5-dimethylthiazol-2-yl)2,5-diphenyltetrazolium bromide) assay after 24-h simulated reperfusion.

The OGD preconditioning experiments consisted of five different treatment groups as follows:

Control (sham-wash): Cells were incubated with the OGD buffer supplemented with $25 \mathrm{~mm}$ glucose for $1 \mathrm{~h}$ in a humidified incubator with normoxia, and then reperfused for $12 \mathrm{~h}$. After $12 \mathrm{~h}$, cells were subjected to 3-h sham OGD, and reperfused for particular periods.

Preconditioning: Cells were subjected to sublethal 1-h OGD and reperfused for $12 \mathrm{~h}$ to induce resistance mechanism before the lethal 3-h OGD.

Lethal OGD: Cells were subjected to 1-h normoxia in the OGD buffer supplemented with $25 \mathrm{~mm}$ glucose, reperfused for $12 \mathrm{~h}$, and then subjected to 3-h OGD and reperfused for particular periods.

Preconditioning + lethal OGD: Cells were preconditioned with 1-h sublethal OGD and reperfused for $12 \mathrm{~h}$, and then subjected to 3-h lethal OGD and reperfused for particular periods.

Fumonisin $\mathrm{B}_{1}+$ preconditioning + lethal OGD: It was the preconditioning + lethal OGD group additionally treated with $50 \mu \mathrm{M}$ of fumonisin $\mathrm{B}_{1}$, a ceramide synthase inhibitor, during the preconditioning period.

Treatment of Neuronal Cultures with Exogenous $\mathbf{C}_{2}$ Ceramide Cell-permeable $\mathrm{C}_{2}$ ceramide was dissolved in $95 \%$ ethanol to make a stock solution with a concentration of $20 \mathrm{~mm}$ and then diluted with serum-free Neurobasal medium to concentrations of 5-50 $\mu \mathrm{m}$ (final ethanol concentration of $0.023-0.23 \%$, respectively). At $7 \mathrm{~d}$ in vitro, cells were washed twice with HEPES-buffered saline and then incubated for $24 \mathrm{~h}$ in serum-free Neurobasal medium containing $\mathrm{C}_{2}$ ceramide at different concentrations $(5-50 \mu \mathrm{M})$ to assess cytotoxicity to primary cultured cortical neuronal cells. Then, to examine the neuroprotective effect of exogenously applied cell-permeable $\mathrm{C}_{2}$ ceramide, it was added to the cultures at a non-cytotoxic concentration of $10 \mu \mathrm{m}$ immediately before the onset of lethal 3-h OGD and remained in the medium during 24-h reperfusion period. After the reperfusion, cell viability was measured by the MTT assay.

Assessment of Cell Injury Neuronal cell injury was observed by phase contrast microscopy and quantified by the MTT assay. Neuron cell viability was estimated by the MTT assay, which is based on the cleavage of yellow MTT salt to purple formazan by mitochondrial dehydrogenase in viable cells. ${ }^{23)}$ In brief, after 24-h simulated reperfusion, the 
medium was removed and the cells were incubated in MTT solution $(0.5 \mathrm{mg} / \mathrm{ml})$ for $2 \mathrm{~h}$ in a humidified $5 \% \mathrm{CO}_{2}$ incubator at $37^{\circ} \mathrm{C}$. After incubation, the medium was aspirated and dimethylsulfoxide was added to dissolve the insoluble purple formazan product into a colored solution which was read at $570 \mathrm{~nm}$ using a THERMOmax microplate reader (Molecular Devices, Menlo Park, CA, U.S.A.). The absorbance of the formazan formed in the sham-washed control cells was taken as $100 \%$ viability.

Sample Preparation and Lipid Extraction for Measurement of Sphingolipids At specific time points after treatments, cells were washed twice with ice-cold phosphatebuffered saline (PBS), harvested and resuspended by ultrasonication in PBS. Aliquots of samples were taken for protein quantification by the Bio-Rad protein assay. Analysis of sphingolipids was done according to the method previously established in our laboratory with little modification. ${ }^{19)}$ In brief, the internal standard $\left(\mathrm{C}_{4: 0}\right.$ ceramide: $0.4 \mu \mathrm{g} / \mathrm{ml}$ methanolic solution) and $0.75 \mathrm{ml}$ of chloroform $/ \mathrm{methanol}$ $(1 / 2, v / v)$ were added to an aliquot of cell suspension in a glass tube and incubated at $48^{\circ} \mathrm{C}$ for $1 \mathrm{~h}$. After adjusting to the neutral $\mathrm{pH}$ with glacial acetic acid, $1 \mathrm{ml}$ of chloroform and $2 \mathrm{ml}$ of water were added to each tube and mixed vigorously. Phase separation was done by centrifugation and the lower organic layer was taken and evaporated to dryness under nitrogen gas in a TurboVap evaporator (Zymark, Hopkinton, MA, U.S.A.). The dried residue was dissolved in $100 \mu \mathrm{l}$ of $0.1 \%$ formic acid in methanol and $10 \mu \mathrm{l}$ were injected into the column. All sphingolipid analysis was normalized to the protein content of the respective cell sample.

Liquid Chromatographic and Mass Spectrometric Condition The HPLC system was performed using a LC10ADvp binary pump system, SIL-10ADvp auto-sampler and CTO-10ASvp oven (Shimadzu, Kyoto, Japan). The analytical column was an XTerra MS $\mathrm{C}_{18}(50 \times 2.1 \mathrm{~mm}$ i.d., $3.5 \mu \mathrm{M}$, Waters, MA, U.S.A.) maintained at $50 \pm 1{ }^{\circ} \mathrm{C}$. The HPLC mobile phases consisted of $5 \mathrm{~mm}$ ammonium formate (pH 4.0)/methanol/tetrahydrofuran (5/2/3, v/v/v) (A) and $5 \mathrm{~mm}$ ammonium formate $(\mathrm{pH} 4.0) / \mathrm{methanol} /$ tetrahydrofuran $(1 / 2 / 7, \mathrm{v} / \mathrm{v} / \mathrm{v})(\mathrm{B})$. A gradient program was set for the HPLC separation at a flow rate of $0.2 \mathrm{ml} / \mathrm{min}$. The initial buffer composition was $90 \%$ of $\mathrm{A}$, then linearly changed to $100 \%$ of $\mathrm{B}$ in $14 \mathrm{~min}$ and maintained for $1 \mathrm{~min}$, followed by reequilibration to the initial condition for $1.2 \mathrm{~min}$. Each run time was $16.2 \mathrm{~min}$.

The HPLC system was coupled on-line to an SCIEX API2000 triple-quadrupole mass spectrometry (Applied Biosystems, Concord, Canada) equipped with a Turbo Ion Spray source. Electrospray ionization was performed in the positive mode with an ionspray voltage of $5400 \mathrm{~V}$. Nitrogen gas was used as the nebulizing, turbo spray and curtain gas with the optimum values set at 40,75 and 40, respectively (arbitrary units). The heated nebulizer temperature was set at $400{ }^{\circ} \mathrm{C}$. The mass spectrometer was operated with low and unit resolution for Q1 and Q3, respectively. The multiple reaction monitoring detection was employed using nitrogen as the collision gas (4 arbitrary unit) with a dwell time of $100 \mathrm{~ms}$ for each transition. The data acquisition was ascertained by the Analyst 1.3.1 program.

Statistical Analysis Data were expressed as the mean S.E.M. All statistical analyses were performed using SPSS and SigmaPlot software (SPSS Inc., Chicago, IL, U.S.A.). Statistical comparison was assessed using the one way analysis of variance test followed by the Duncan's post-hoc test, and $p$-value $<0.05$ was considered statistically significant. Each experiment was performed at least three times in different cultures.

\section{RESULTS}

Ischemic Tolerance by Preconditioning with OxygenGlucose Deprivation To explore the signaling pathways and mediators of ischemic tolerance, we established a preconditioning model system by OGD in 100-mm dish with near pure $6-8 \mathrm{~d}$ in vitro primary cultured cortical neurons of rats. The morphological analysis of cortical neurons subjected to lethal 3-h OGD showed extensive neuronal cell damage with most of the neuronal processes degenerated after 24-h reperfusion (Fig. 1c). Neurons which were preconditioned with sublethal 1-h OGD were more resistant to subsequent lethal 3-h OGD treatment showing most of the neuronal processes intact (Fig. 1d). Quantitative analyses of cell injuries by simulated in vitro ischemia were performed using the MTT assay (Fig. 1e). It was found that only $45.3 \%$ of the neurons survived after only lethal 3-h OGD treatment when

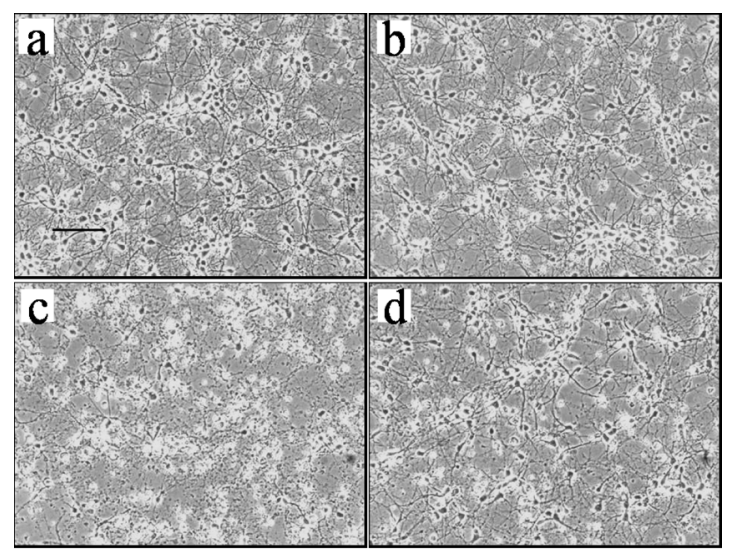

e

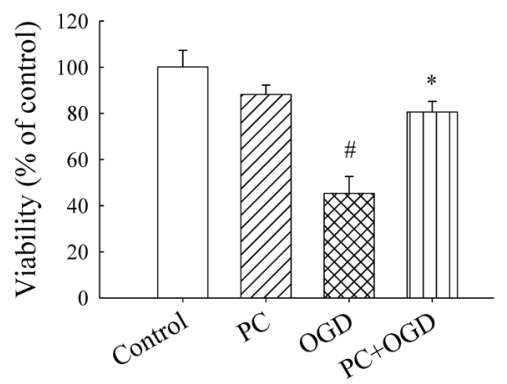

Fig. 1. Morphological Photographs and Cell Viability Measurement Showing Neuroprotective Effect of Preconditioning (PC) with Sublethal 1-h Oxygen-Glucose Deprivation (OGD) on Cell Death Caused by Lethal 3-h OGD in Primary Cultured Cortical Neurons of Rats

At $6-7 \mathrm{~d}$ in vitro, primary cultured cortical neurons were preconditioned with sublethal 1-h OGD followed by 12-h normoxia and then exposed to lethal 3-h OGD. Phase contrast microscopic photographs of primary cultured cortical neurons subjected to sham-wash (control, a), PC (b), OGD (c), and PC + OGD (d) treatments, were taken following 24-h reperfusion after each treatment. The scale bar is $50 \mu \mathrm{m}$. Quantitative cell viability was also measured by the MTT assay (e) following 24-h reperfusion after each treatment. Values are given as mean \pm S.E.M. of at least four independent experiments. ${ }^{\#} p<0.01$ compared with the control group; $* p<0.01$ compared with the OGD group. Control, $24 \mathrm{~h}$ after sham-wash; PC, 1-h OGD and 24-h reperfusion; OGD, 3-h OGD and 24-h reperfusion; PC+OGD, 1-h OGD and 12-h reperfusion and then 3-h OGD and 24-h reperfusion. 
Table 1. Temporal Profile of Various Ceramide Levels during Reperfusion Period following Preconditioning with Sublethal 1-h Oxygen-Glucose Deprivation (OGD)

\begin{tabular}{|c|c|c|c|c|c|c|c|}
\hline Samples & $\mathrm{C}_{16: 0}$ Cer & $\mathrm{C}_{18: 0}$ Cer & $\mathrm{C}_{18: 1}$ Cer & $\mathrm{C}_{20: 0}$ Cer & $\mathrm{C}_{24: 0} \mathrm{Cer}$ & $\mathrm{C}_{24: 1}$ Cer & Total \\
\hline Control & $396.4 \pm 35.5$ & $1949.8 \pm 134.7$ & $33.1 \pm 4.6$ & $169.5 \pm 15.4$ & $38.5 \pm 5.0$ & $197.2 \pm 28.3$ & $2784.6 \pm 216.1$ \\
\hline $\mathrm{PC}+2-\mathrm{h} \mathrm{R}$ & $433.6 \pm 23.3$ & $2189.6 \pm 134.0$ & $37.3 \pm 3.2$ & $183.3 \pm 17.3$ & $42.2 \pm 3.3$ & $238.0 \pm 28.5$ & $3124.0 \pm 204.5$ \\
\hline $\mathrm{PC}+6-\mathrm{h} \mathrm{R}$ & $515.2 \pm 34.2 *$ & $2638.1 \pm 170.4^{*}$ & $49.5 \pm 6.5$ & $241.3 \pm 25.7^{*}$ & $58.0 \pm 6.9^{*}$ & $330.2 \pm 39.2 *$ & $3832.3 \pm 266.0 *$ \\
\hline $\mathrm{PC}+12-\mathrm{h} \mathrm{R}$ & $508.9 \pm 24.8^{*}$ & $2654.9 \pm 139.5^{*}$ & $49.6 \pm 5.0$ & $237.8 \pm 18.3^{*}$ & $45.5 \pm 4.0$ & $292.1 \pm 29.6$ & $3788.7 \pm 200.8 *$ \\
\hline
\end{tabular}

Six different ceramide (Cer) levels (pmol/mg protein) were measured at the indicated time of reperfusion following preconditioning (PC) with sublethal 1-h OGD in primary cultured cortical neurons according to our previously published LC-MS/MS method. ${ }^{19)}$ Values are given as mean \pm S.E.M. of at least three independent experiments. $* p<0.05$ compared with the control group. $\mathrm{R}$ denotes reperfusion.

Table 2. Temporal Profile of Various Sphingomyelin Levels during Reperfusion Period following Preconditioning with Sublethal 1-h Oxygen-Glucose Deprivation (OGD)

\begin{tabular}{lcccc}
\hline \hline Samples & $\mathrm{SM} \mathrm{C}_{16: 0}$ & $\mathrm{SM} \mathrm{C}_{18: 0}$ & $\mathrm{SM} \mathrm{C}_{24: 0}$ & Total \\
\hline Control & $605.6 \pm 48.6$ & $4816.8 \pm 323.7$ & $55.7 \pm 3.8$ & $5478.1 \pm 371.5$ \\
PC+2-h R & $602.0 \pm 55.4$ & $4780.4 \pm 348.6$ & $58.5 \pm 7.2$ & $5441.0 \pm 396.6$ \\
PC+6-h R & $703.0 \pm 79.7$ & $5608.9 \pm 681.4$ & $72.1 \pm 9.5$ & $6384.0 \pm 768.7$ \\
PC+12-h R & $610.6 \pm 57.9$ & $4748.3 \pm 464.7$ & $61.4 \pm 8.4$ & $5420.4 \pm 524.5$
\end{tabular}

Three different sphingomyelin (SM) levels (pmol/mg protein) were measured at the indicated time of reperfusion following preconditioning (PC) with sublethal 1-h OGD in primary cultured cortical neurons according to our previously published LC-MS/MS method. ${ }^{19)}$ Values are given as mean \pm S.E.M. of at least three independent experiments. $\mathrm{R}$ denotes reperfusion.

measured after 24-h reperfusion. On the other hand, preconditioning of primary cultured cortical neurons with sublethal 1-h OGD produced robust neuroprotection against cell death induced by lethal 3-h OGD imposed $12 \mathrm{~h}$ after preconditioning, significantly increasing cell viability to $80.5 \%$ compared with the only 3-h OGD treatment group (Fig. 1e). The level of cell survival in the preconditioning-induced ischemic tolerant group was similar to that observed in the only sublethal 1-h OGD treatment group. No significant loss of cell viability was observed in the sublethal 1-h OGD treatment group (Figs. 1b, e).

Increases in Ceramide Levels by Preconditioning with Oxygen-Glucose Deprivation Levels of multiple sphingolipids were simultaneously measured from scarce primary cultured cortical neuron cell samples using sensitive and specific LC-MS/MS technique. The ceramide species in primary cultured cortical neuronal cells were characterized as long chain, including $\mathrm{C}_{16: 0}$ Cer, $\mathrm{C}_{18: 0}$ Cer, $\mathrm{C}_{18: 1}$ Cer, $\mathrm{C}_{20: 0}$ Cer, $\mathrm{C}_{24: 0}$ Cer and $\mathrm{C}_{24: 1}$ Cer. Notably, the $\mathrm{C}_{18: 0}$ ceramide levels were much higher (about $70 \%$ of the total) than any other ceramide species (Table 1) in primary cultured cortical neurons because the gene responsible for this ceramide is predominantly expressed in the brain. ${ }^{24)}$ The total baseline ceramide content was $2784.6 \pm 216.1 \mathrm{pmol} / \mathrm{mg}$ protein. It has been reported that neuronal cells contain a higher level of ceramide than other types of cells. ${ }^{25)}$ In addition, long chain-containing sphingomyelin (SM) species, including $\mathrm{SM} \mathrm{C}_{16: 0}, \mathrm{SM} \mathrm{C}_{18: 0}$ and $\mathrm{SM} \mathrm{C}_{24: 0}$, could be measured in primary cultured cortical neurons of rats (Table 2). Our result is in agreement with previously published report that $\mathrm{SM} \mathrm{C}_{16: 0}$ and $\mathrm{SM} \mathrm{C}_{18: 0}$ are main sphingomyelins in cultured neurons of rats. ${ }^{26}$ )

To examine the involvement of sphingolipids in ischemic tolerance paradigm, sphingolipid quantifications were performed at 2, 6 and $12 \mathrm{~h}$ after sublethal 1-h OGD preconditioning. As shown in Fig. 2 and Table 1, there were no signif-

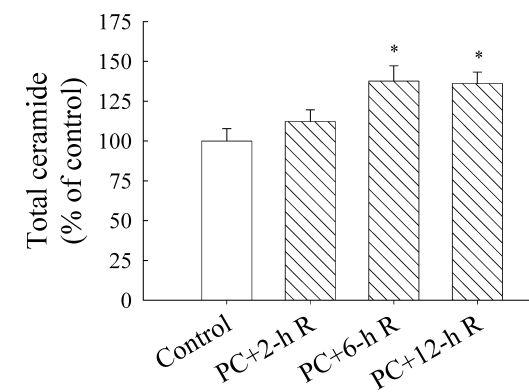

Fig. 2. Temporal Profile of Total Ceramide Contents during Reperfusion Period following Preconditioning with Sublethal 1-h Oxygen-Glucose Deprivation (OGD)

Primary culture of cortical neurons of rats were grown in 100-mm tissue culture dishes and, at $6 \mathrm{~d}$ in vitro, were preconditioned with sublethal 1-h OGD. Ceramide levels in primary cultured cortical neurons were measured by means of LC-MS/MS technique at the indicated reperfusion time after sublethal preconditioning. The total ceramide contents of the sham-washed control group measured at each reperfusion time point after preconditioning were pooled and expressed as $100 \%$. Values are given as mean \pm S.E.M. of at least three independent experiments; $* p<0.05$ compared with the control group. Control, sham-wash; PC, 1-h OGD.

icant changes in cellular ceramide levels at 2-h reperfusion time after the preconditioning. However, the levels of most ceramide species were significantly increased at 6-h reperfusion time after the preconditioning and the increases were sustained up to 12-h reperfusion time (Table 1). In particular, preconditioning treatment resulted in significant increases in $\mathrm{C}_{16: 0}$ Cer, $\mathrm{C}_{18: 0}$ Cer, $\mathrm{C}_{20: 0}$ Cer, $\mathrm{C}_{24: 0}$ Cer, $\mathrm{C}_{24: 1}$ Cer and the total ceramide contents compared with the sham-washed control group (Table 1, Fig. 2). In contrast, levels of the sphingomyelin species in primary cultured cortical neurons, including $\mathrm{SM} \mathrm{C}_{16: 0}, \mathrm{SM} \mathrm{C}_{18: 0}$ and $\mathrm{SM} \mathrm{C}_{24: 0}$, were not significantly altered at any reperfusion time points tested after the preconditioning, indicating that the increases in ceramide levels did not result from sphingomyelin hydrolysis (Table 2).

When the ceramide and sphingomyelin levels of neuronal cells subjected to lethal 3-h OGD were measured at 1, 6 and 24-h reperfusion time points, there were no significant differences in the total ceramide and sphingomyelin levels of the lethal OGD group or the preconditioning+lethal OGD group, compared with those of the sham-washed control group (Fig. 3).

Abolishment of Preconditioning-Induced Ischemic Tolerance by Pharmacological Blockade of de Novo Ceramide Synthesis during Preconditioning Period To examine the role of increased ceramide levels in preconditioning-induced ischemic tolerance, primary cultured cortical neurons were incubated with $50 \mu \mathrm{M}$ of fumonisin $\mathrm{B}_{1}$, a ceramide synthase inhibitor, during the preconditioning period. 

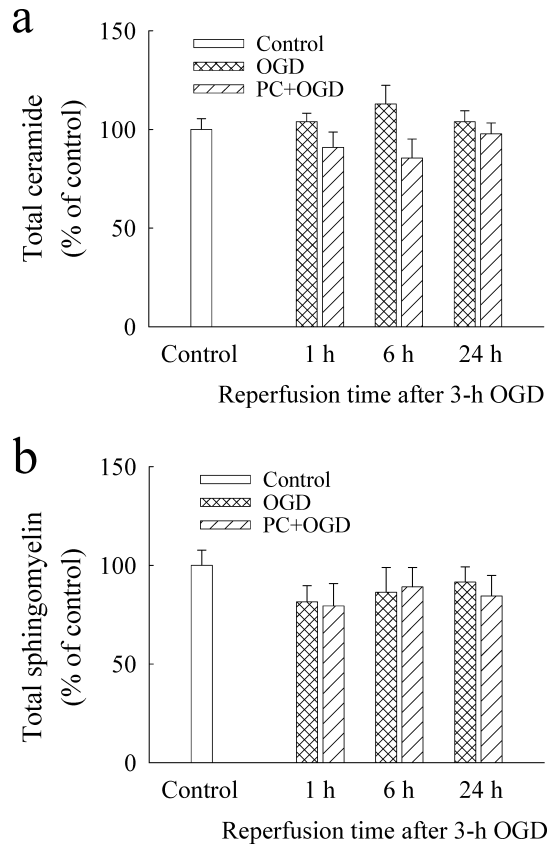

Fig. 3. Temporal Profile of Total Ceramide and Total Sphingomyelin Levels during Reperfusion Period following Lethal 3-h Oxygen-Glucose Deprivation (OGD)

Primary culture of cortical neurons of rats were grown in 100-mm tissue culture dishes and, at $7 \mathrm{~d}$ in vitro, were subjected to lethal 3-h OGD. Ceramide (a) and sphingomyelin (b) levels in primary cultured cortical neurons were measured by means of LC-MS/MS technique at the indicated reperfusion time following lethal 3-h OGD. The total ceramide and total sphingomyelin contents of the sham-washed control group measured at each reperfusion time point after lethal 3-h OGD were pooled and expressed as $100 \%$ ceramide and $100 \%$ sphingomyelin, respectively. Values are given as mean \pm S.E.M. of at least three independent experiments. Control, sham-wash; OGD, 3 h OGD; PC+OGD, 1-h OGD 12-h reperfusion and then 3-h OGD.

At the concentration used, fumonisin $\mathrm{B}_{1}$ itself did not significantly affect primary cultured cortical neuron cell viability (Figs. 4b, f), which is consistent with previously published report. ${ }^{27)}$ However, treatment of neuronal cells with fumonisin $\mathrm{B}_{1}$ during the preconditioning period completely abolished the preconditioning-induced ischemic tolerance (Figs. 4e, f).

Neuroprotective Effect of a Non-cytotoxic Concentration of Exogenous $\mathrm{C}_{2}$ Ceramide against Lethal OxygenGlucose Deprivation Application of relatively low concentrations of cell-permeable $\mathrm{C}_{2}$ ceramide $(5,10 \mu \mathrm{M})$ to primary cultured cortical neurons for $24 \mathrm{~h}$ had no cytotoxic effects on cell viability when measured by the MTT assay (Fig. 5). On the other hand, it was found that application of higher concentrations $(20,40,50 \mu \mathrm{M})$ of $\mathrm{C}_{2}$ ceramide for $24 \mathrm{~h}$ produced dose-dependent decreases in cell viability. Since $10 \mu \mathrm{M}$ of exogenously applied $\mathrm{C}_{2}$ ceramide was shown to be noncytotoxic to primary cultured cortical neurons, we examined whether this concentration of $\mathrm{C}_{2}$ ceramide could produce neuroprotective effect against the lethal OGD. Interestingly, the presence of $10 \mu \mathrm{M}$ of $\mathrm{C}_{2}$ ceramide during and after lethal OGD produced robust neuroprotection with increasing cell viability from 41.5 to $78.3 \%$ when measured $24 \mathrm{~h}$ after reperfusion (Fig. 6). The extent of neuroprotection produced by the non-cytotoxic concentration of exogenous $\mathrm{C}_{2}$ ceramide was similar to that observed by the sublethal OGD preconditioning (Figs. 1e, 6).
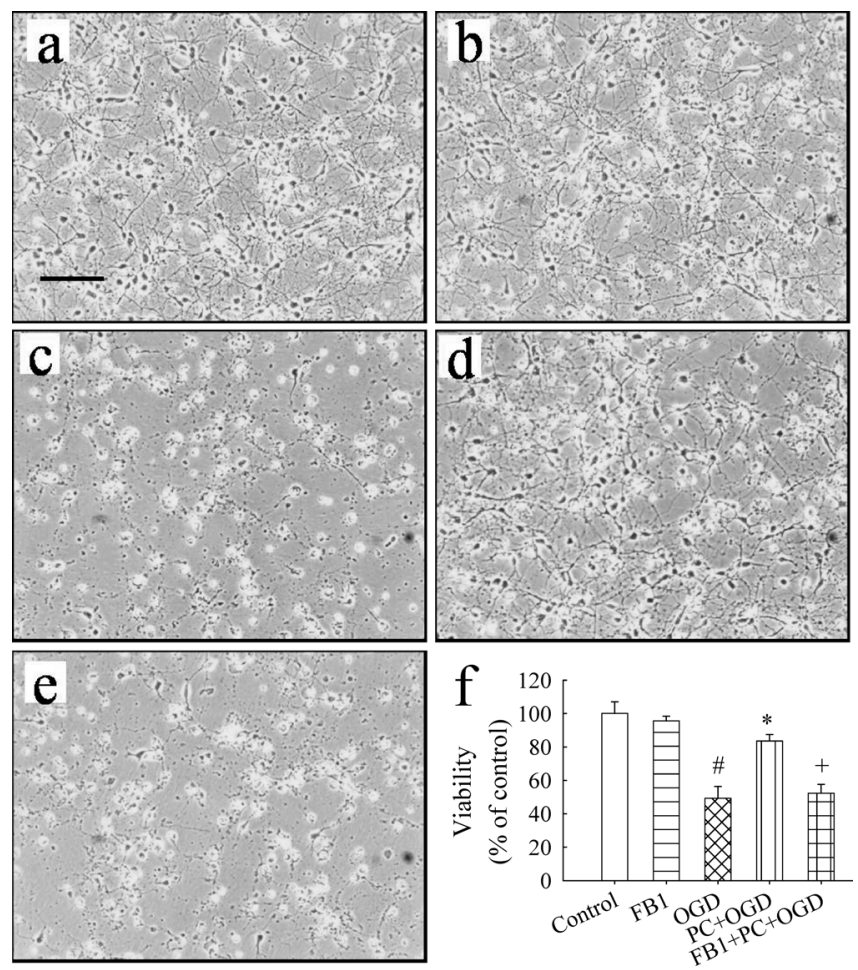

Fig. 4. Morphological Photographs and Cell Viability Measurement Showing Blockade of Preconditioning-Induced Ischemic Tolerance by Fumonisin $\mathrm{B}_{1}$ in Primary Cultured Cortical Neurons of Rats

Preconditioning (PC) with sublethal 1-h oxygen-glucose deprivation (OGD) resulted in tolerance to cell death caused by subsequent lethal 3-h OGD in primary cultured cortical neurons of rats, which was blocked by the treatment of neurons with fumonisin $B_{1}$ $\left(\mathrm{FB}_{1}\right)$, a ceramide synthase inhibitor, during the preconditioning period. Phase contrast microscopic photographs of primary cultured cortical neurons subjected to sham-wash (control, a), $\mathrm{FB}_{1}$ (b), OGD (c), PC+OGD (d), and $\mathrm{FB}_{1}+\mathrm{PC}+\mathrm{OGD}$ (e) treatments, were taken following 24-h reperfusion after each treatment. The scale bar is $50 \mu \mathrm{m}$. Quantitative cell viability was also measured by the MTT assay (f) following 24-h reperfusion after each treatment. Values are given as mean \pm S.E.M. of at least four independent experiments. ${ }^{2} p<0.01$ compared with the control group; $* p<0.01$ compared with the OGD group; ${ }^{+} p<0.01$ compared with the $\mathrm{PC}+\mathrm{OGD}$ group. Control, $24 \mathrm{~h}$ after shamwash; OGD, 3-h OGD and 24-h reperfusion; PC+OGD, 1-h OGD and 12-h reperfusion and then 3-h OGD and 24-h reperfusion; $\mathrm{FB}_{1}, 50 \mu \mathrm{M}$ of fumonisin $\mathrm{B}_{1}$.

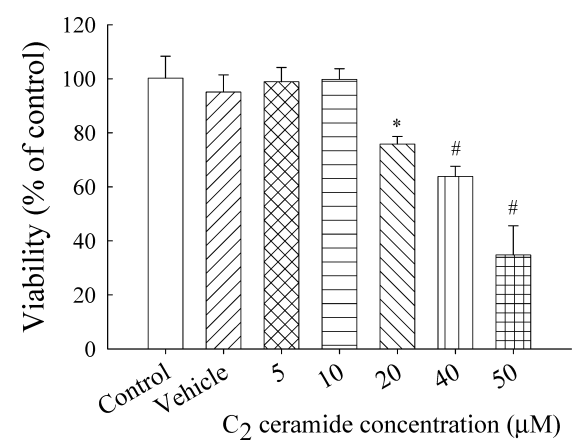

Fig. 5. Cell Viability Measurement Showing Dose-Dependent Cytotoxicity of Exogenous Cell-Permeable $\mathrm{C}_{2}$ Ceramide to Primary Cultured Cortical Neurons of Rats

At $7 \mathrm{~d}$ in vitro, primary cultured cortical neurons were treated for $24 \mathrm{~h}$ with cell-permeable $\mathrm{C}_{2}$ ceramide at the indicated concentrations in serum free Neurobasal medium and then cell viability was measured by the MTT assay. Values are given as mean \pm S.E.M. of at least three independent experiments. $* p<0.05$, $\# p<0.01$ compared with the vehicle-treated group. Control, $24 \mathrm{~h}$ after sham-wash; vehicle, $0.23 \%$ ethanol.

\section{DISCUSSION}

Ischemic stress induces both harmful and protective responses and the balance between these two determines the 


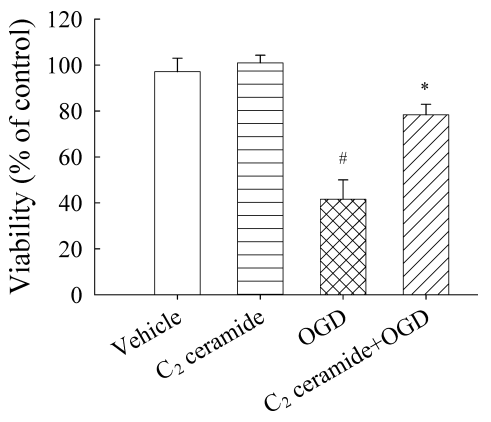

Fig. 6. Cell Viability Measurement Showing Neuroprotective Effect of a Non-cytotoxic Concentration of Exogenous Cell-Permeable $\mathrm{C}_{2}$ Ceramide against Lethal 3-h Oxygen-Glucose Deprivation (OGD) in Primary Cultured Cortical Neurons of Rats

At $7 \mathrm{~d}$ in vitro, neuronal cultures were subjected to lethal 3-h OGD in the presence or absence of $10 \mu \mathrm{M}$ of $\mathrm{C}_{2}$ ceramide. Quantitative cell viability was measured by the MTT assay following 24-h reperfusion after each treatment. Values are given as mean \pm S.E.M. of at least four independent experiments. ${ }^{\#} p<0.01$ compared with the vehicle-treated group; $* p<0.01$ compared with the OGD group. Vehicle, $0.046 \%$ ethanol; OGD, 3-h OGD and 24-h reperfusion; $\mathrm{C}_{2}$ ceramide, $10 \mu \mathrm{M}$ of $\mathrm{C}_{2}$ ceramide.

cellular fate. ${ }^{28)}$ If the stress is sublethal i.e. below the threshold of damage, protective mechanisms prevail. When another stress is reapplied at the peak of the stress-controlling period, cells show better tolerance. This phenomenon is known as stress adaptation or preconditioning, in which sublethal stress induces an adaptive response to subsequent lethal stress. But, the molecular mechanism(s) that is switched on during the sublethal preconditioning period or the early signaling mediators that trigger the resistance mechanism to ischemic stress are not well defined. The present results indicate that ceramide acts as an early player in the induction of ischemic tolerance caused by preconditioning with sublethal oxygenglucose deprivation in primary cultured cortical neurons of rats.

The versatile roles of ceramide in neuronal physiology and pathology have drawn attention due to the apparent contradictory results that have been reported with respect to the effects of ceramide on cell survival and death. In general, low concentrations of ceramide were found to promote cell survival in the hippocampal, ${ }^{29)}$ cerebellar Purkinje $^{30)}$ and sympathetic $^{31)}$ neurons, whereas higher concentrations of ceramide were found to induce cell death in the hippocampal, ${ }^{29)}$ corti$\mathrm{cal}^{32)}$ and mesencephalic ${ }^{33)}$ neurons. The studies that correlated ceramide with neurodegeneration were of higher levels of ceramide accumulation. ${ }^{8,34)}$ The bimodal roles of ceramide in different cellular processes can be explained by concentration, ${ }^{18)}$ cell types, ${ }^{17)}$ sub-cellular localization, ${ }^{35)}$ microenvironment, ${ }^{36)}$ etc.

In the present study, using primary cultured cortical neurons of rats, significant increases in ceramide levels were observed during 6 to $12 \mathrm{~h}$ of reperfusion period following sublethal OGD treatment. The time course of ceramide increment is consistent with the hypothesis that ceramide plays a role in the early stage of the tolerance process. Increases in ceramide levels by different preconditioning stimuli such as lipopolysaccharide, ${ }^{37)}$ exercise, ${ }^{9,10)}$ hypoxia ${ }^{16}$ and tumor necrosis factor- $\alpha,{ }^{38)}$ are in accordance with our observation. Moreover, the present results clearly showed that the increases in ceramide levels during the preconditioning paradigm are critical for the preconditioning-induced ischemic tolerance. But the molecular mechanism(s) of how ceramide mediates ischemic tolerance remains to be elucidated. In this context, Chen et al. (2001) reported that exogenous ceramide reduced the infarct size in focal cerebral ischemia with an upregulation of $\mathrm{Bcl}-2$ and a reduction of apoptotic cells. ${ }^{15}$ ) Song and de Chaves (2003) showed that both exogenously added and de novo pathway-generated ceramides were antiapoptotic. ${ }^{39)}$ They demonstrated that ceramide activated the phosphatidylinositol 3-kinase (PI3K)/Akt pathway although it was not concluded whether the activation was caused via receptor tyrosine kinase or via non-receptor tyrosine kinase or directly to PI3K. MacPhee and Barker (1999) reported that ceramide treatment increased trkA activation by altering the plasma membrane and subsequent activation of PI3K and extracellular signal-regulated kinase $1 / 2$ in PC12 cells. $^{40)}$ Hanna et al. (1999) showed that ceramide could activate the PI3K/Akt pathway through mechanisms dependent on nonreceptor tyrosine kinase activity in rat2 fibroblasts. ${ }^{41)}$ In this regard, we have also found that the PI3K/Akt pathway is involved in ischemic tolerance induced by sublethal OGD preconditioning (unpublished observation). Therefore, it is suggested that there is an interaction between the PI3K/Akt pathway and ceramide in OGD preconditioning induced ischemic tolerance. In addition, several other postulations, such as induction of Mn-SOD transcription ${ }^{42)}$ and regulation of transcription factor NF- $\left.\kappa \mathrm{B},{ }^{43}\right)$ have also been put forward to try to explain how ceramide promotes cellular defense mechanisms.

Ceramide can be produced in the cells through sphingomyelin hydrolysis or by de novo synthesis. In the present study, concomitant with the increment of ceramide levels, sphingomyelin contents in cultured neurons were not altered during the preconditioning period, indicating that ceramide levels were increased by de novo synthesis. It has been demonstrated that the ceramide generation by sphingomyelin hydrolysis takes a short time, just minutes, ${ }^{44)}$ whereas de novo synthesis needs several hours. ${ }^{45)}$ Our finding that increased ceramide levels were observed at approximately $6 \mathrm{~h}$ of reperfusion period further supported that it occurred via de novo synthetic pathway. To examine the functional role of increased ceramide levels in ischemic tolerance, the neuronal cells were treated with a ceramide synthase inhibitor, fumonisin $\mathrm{B}_{1}$, at a concentration of $50 \mu \mathrm{M}$ known to completely inhibit ceramide synthase during the preconditioning period. $\left.{ }^{46}\right)$ Surprisingly, the treatment with fumonisin $\mathrm{B}_{1}$ completely abolished subsequent ischemic tolerance. The results clearly demonstrate that ceramide produced via de novo synthetic pathway plays a critical role in the induction of ischemic tolerance. However, the regulatory mechanism on ceramide synthase by preconditioning remains to be elucidated.

In addition, to examine the sphingolipid regulation in neuronal cell death induced by severe OGD, the ceramide and sphingomyelin contents were measured after subjecting the neurons to lethal 3-h OGD. However, no regulation of ceramide and sphingomyelin was observed in the severe OGD paradigm. Although some in vivo ischemic studies ${ }^{8,34,44)}$ indicated the relationship between ceramide accumulation and cell death, our results showed that the ceramide pathway was not involved in in vitro lethal OGD-induced neuronal cell death. It may be possible that differences in ischemic models and severity dictate ceramide generation and cell death pathways. Moreover, it was found that the increased levels of 
total ceramide in neurons preconditioned with sublethal OGD returned to the basal levels when measured at $1 \mathrm{~h}$ of reperfusion following subsequent lethal 3-h OGD. A possibility is that it might be resulted from cellular metabolism of ceramides within the 4-h interval between the 1-h OGD+12$\mathrm{h}$ reperfusion point and the $3-\mathrm{h}$ OGD $+1-\mathrm{h}$ reperfusion point of ceramide analysis.

It can be noted that most studies related to biological functions of ceramide were done with exogenously applied short chain cell-permeable ceramides. In the present study, it was demonstrated that small (approximately 1.4-fold over the sham-washed control) increases in endogenous ceramide levels induced by sublethal OGD and their presence at the onset of lethal OGD played an important role in neuroprotection against subsequent lethal OGD. Moreover, it was shown that exogenous application of a relatively low concentration $(10 \mu \mathrm{M})$ of cell-permeable $\mathrm{C}_{2}$ ceramide at the onset of lethal OGD produced neuroprotection against lethal OGD in primary cultured cortical neurons, while $\mathrm{C}_{2}$ ceramide at concentrations greater than $10 \mu \mathrm{M}$ showed dose-dependent cytotoxicity. The extent of neuroprotection produced by the non-cytotoxic concentration of exogenous $\mathrm{C}_{2}$ ceramide was similar to that observed by the sublethal OGD preconditioning, further supporting our hypothesis that ceramide was involved in ischemic tolerance induced by sublethal OGD preconditioning in primary cultured cortical neurons of rats. However, there is still controversy of using short chain ceramides because ceramide species found in neuronal cells consist of long fatty acid chain. ${ }^{26}$ Another point to be considered is whether the concentration of ceramide used to promote cell survival or to induce cell death in cultures is relevant to the endogenous levels found in physiological or pathological conditions. Although intracellular ceramide levels may vary with cell types, ${ }^{25}$ typical picomole to nanomole range regulations of ceramide warrants further thought of using micromolar levels of exogenous ceramide. In this regard, ischemic preconditioning could be a good choice of models to study the regulation of endogenous ceramide levels and their (patho)physiological roles.

In summary, the present study demonstrate that preconditioning-induced ischemic tolerance is associated with increases in ceramide levels during sublethal OGD preconditioning, whereas ceramide regulation is not associated with lethal OGD-induced neuronal cell death. Furthermore, the results indicate that ceramide levels increased via de novo synthesis during the preconditioning period play an important role in mediating tolerance to subsequent lethal ischemic stress in primary cultured cortical neurons of rats. Further exploration of the signaling pathway(s) of ceramide in ischemic tolerance paradigm may broaden our insight into therapeutic interventions for ischemic stroke.

Acknowledgements This research was supported by a grant (PF06216-00) from Plant Diversity Research Center of 21 st Century Frontier Research program funded by Ministry of Science and Technology of Korean Government.

\section{REFERENCES}

1) Bruer U., Weih M. K., Isaev N. K., Meisel A., Ruscher K., Bergk A., Trendelenburg G., Wiegand F., Victorov I. V., Dirnagl U., FEBS Lett., 414, 117-121 (1997).
2) Kapinya K. J., Acta Physiol. Hung., 92, 67-92 (2005).

3) Colombaioni L., Garcia-Gil M., Brain Res. Rev., 46, 328-355 (2004).

4) Goswami R., Dawson G., J. Neurosci. Res., 60, $141-149$ (2000).

5) Hannun Y. A., Luberto C., Trends Cell Biol., 10, 73-80 (2000).

6) Han X. L., Holtzman D. M., McKeel D. W., Kelley J., Morris J. C., J. Neurochem., 82, 809-818 (2002).

7) Mikati M. A., Abi-Habib R. J., El Sabban M. E., Dbaibo G. S., Kurdi R. M., Kobeissi M., Farhat F., Asaad W., Epilepsia, 44, 282-291 (2003).

8) Kubota M., Narita K., Nakagomi T., Tamura A., Shimasaki H., Ueta N., Yoshida S., Neurol. Res., 18, 337-341 (1996).

9) Blachnio-Zabielska A., Baranowski M., Zabielski P., Gorski J., J. Cell. Biochem., 105, 776-784 (2008).

10) Helge J. W., Dobrzyn A., Saltin B., Gorski J., Exp. Physiol., 89, 119 127 (2004).

11) Ding Y., Li J., Luan X., Ding Y. H., Lai Q., Rafols J. A., Phillis J. W. Clark J. C., Diaz F. G., Neuroscience, 124, 583-591 (2004).

12) Ang E. T., Wong P. T., Moochhala S., Ng Y. K., Neuroscience, 118, $335-345$ (2003)

13) Posse de Chaves E. I., Biochim. Biophys. Acta, 1758, 1995-2015 (2006).

14) Goodman Y., Mattson M. P., J. Neurochem., 66, 869-872 (1996).

15) Chen Y., Ginis I., Hallenbeck J. M., J. Cereb. Blood Flow Metab., 21, $34-40$ (2001).

16) Liu J., Ginis T., Spatz M., Hallenbeck J. M., Am. J. Physiol. Cell Physiol., 278, C144-C153 (2000).

17) Casaccia-Bonnefil P., Aibel L., Chao M. V., J. Neurosci. Res., 43, 382-389 (1996).

18) Irie F., Hirabayashi Y., J. Neurosci. Res., 54, 475-485 (1998).

19) Yoo H. H., Son J., Kim D. H., J. Chromatogr. B, 843, 327-333 (2006).

20) Brewer G. J., J. Neurosci. Res., 42, 674-683 (1995).

21) Lautenschlager M., Onufriev M. V., Gulyaeva N. V., Harms C., Freyer D., Sehmsdorf U., Ruscher K., Moiseeva Y. V., Arnswald A., Victorov I., Dirnagl U., Weber J. R., Hortnagl H., Neuroscience, 97, 383-393 (2000).

22) Goldberg M. P., Choi D. W., J. Neurosci., 13, 3510-3524 (1993).

23) Hansen M. B., Nielsen S. E., Berg K., J. Immunol. Methods, 119 203-210 (1989).

24) Jiang J. C., Kirchman P. A., Zagulski M., Hunt J., Jazwinski S. M., Genome Res., 8, 1259-1272 (1998).

25) Riboni L., Bassi R., Prinetti A., Viani P., Tettamanti G., Biochem. J., 338, 147-151 (1999).

26) Valsecchi M., Mauri L., Casellato R., Prioni S., Loberto N., Prinetti A., Chigorno V., Sonnino S., J. Lipid Res., 48, 417-424 (2007).

27) Osuchowski M. F., Sharma R. P., Neurotoxicology, 26, 981-992 (2005).

28) Dirnagl U., Simon R. P., Hallenbeck J. M., Trends Neurosci., 26, 248 254 (2003).

29) Mitoma J., Ito M., Furuya S., Hirabayashi Y., J. Neurosci. Res., 51, $712-722$ (1998).

30) Furuya S., Mitoma J., Makino A., Hirabayashi Y., J. Neurochem., 71, 366-377 (1998)

31) Ito A., Horigome K., J. Neurochem., 65, 463-466 (1995).

32) Willaime S., Vanhoutte P., Caboche J., Lemaigre-Dubreuil Y., Mariani J., Brugg B., Eur. J. Neurosci., 13, 2037-2046 (2001).

33) Brugg B., Michel P. P., Agid Y., Ruberg M., J. Neurochem., 66, 733 739 (1996).

34) Herr I., Martin-Villalba A., Kurz E., Roncaioli P., Schenkel J., Cifone M. G., Debatin K. M., Brain Res., 826, 210-219 (1999).

35) Perry R. J., Ridgway N. D., J. Lipid Res., 45, 164-173 (2004).

36) Kolesnick R., Fuks Z., J. Exp. Med., 181, 1949-1952 (1995).

37) Zimmermann C., Ginis I., Furuya K., Klimanis D., Ruetzler C., Spatz M., Hallenbeck J. M., Brain Res., 895, 59-65 (2001).

38) Ginis I., Schweizer U., Brenner M., Liu J., Azzam N., Spatz M., Hallenbeck J. M., Am. J. Physiol. Cell Physiol., 276, C1171-C1183 (1999).

39) Song M. S., de Chaves E. I. P., Neuropharmacology, 45, 1130-1150 (2003).

40) MacPhee I., Barker P. A., J. Neurochem., 72, 1423-1430 (1999).

41) Hanna A. N., Chan E. Y. W., Xu J., Stone J. C., Brindley D. N., J. Biol. Chem., 274, 12722-12729 (1999).

42) Pahan K., Dobashi K., Ghosh B., Singh I., J. Neurochem., 73, 513 520 (1999).

43) Ginis I., Jaiswal R., Klimanis D., Liu H., Greenspon J., Hallenbeck J. M., J. Cereb. Blood Flow Metab., 22, 142-152 (2002).

44) Nakane M., Kubota M., Nakagomi T., Tamura A., Hisaki H., Shimasaki H., Ueta N., Neurosci. Lett., 296, 89-92 (2000).

45) Bose R., Verheij M., Haimovitzfriedman A., Scotto K., Fuks Z., Kolesnick R., Cell, 82, 405-414 (1995).

46) Merrill A. H., Jr., van Echten G., Wang E., Sandhoff K., J. Biol. Chem., 268, 27299-27306 (1993). 